\title{
A unified accretion-ejection paradigm for black hole X-ray binaries
}

\section{The dynamical constituents}

\author{
J. Ferreira ${ }^{1}$, P.-O. Petrucci ${ }^{1}$, G. Henri ${ }^{1}$, L. Saugé ${ }^{1,2}$, and G. Pelletier ${ }^{1}$ \\ 1 Laboratoire d'Astrophysique, Observatoire de Grenoble, BP 53, 38041 Grenoble Cedex 9, France \\ e-mail: Jonathan.Ferreira@obs.ujf-grenoble.fr \\ 2 Institut de Physique Nucléaire de Lyon, 43 Bd 11 novembre 1918, 69622 Villeurbanne Cedex, France
}

Received 12 January 2005 / Accepted 19 October 2005

\section{ABSTRACT}

We present a new picture for the central regions of Black Hole X-ray Binaries. In our view, these central regions have a multi-flow configuration which consists in (1) an outer standard accretion disc down to a transition radius $r_{J}$; (2) an inner magnetized accretion disc below $r_{J}$ driving (3) a non relativistic self-collimated electron-proton jet surrounding, when adequate conditions for pair creation are met; (4) a ultra relativistic electron-positron beam.

This accretion-ejection paradigm provides a simple explanation to the canonical spectral states, from radio to $\mathrm{X} / \gamma$-rays, by varying the transition radius $r_{J}$ and disc accretion rate $\dot{m}$ independently. Large values of $r_{J}$ correspond to the Quiescent state for low $\dot{m}$ and the Hard state for larger $\dot{m}$. These states are characterized by the presence of a steady electron-proton MHD jet emitted by the disc below $r_{J}$. The hard X-ray component is expected to form at the jet basis. When $r_{I}$ becomes smaller than the marginally stable orbit $r_{i}$, the whole disc resembles a standard accretion disc with no jet, characteristic of the Soft state. Intermediate states correspond to situations where $r_{J} \gtrsim r_{i}$. At large $\dot{m}$, an unsteady pair cascade process is triggered within the jet axis, giving birth to flares and ejection of relativistic pair blobs. This would correspond to the luminous intermediate state, sometimes referred to as the Very High state, with its associated superluminal motions.

The variation of $r_{J}$ independently of $\dot{m}$ is a necessary ingredient in this picture. It arises from the presence of a large scale vertical magnetic field threading the disc. Features such as possible hysteresis and the presence of quasi-periodic oscillations would naturally fit within this new framework.

Key words. black hole physics - accretion, accretion disks - magnetohydrodynamics (MHD) - ISM: jets and outflows - X-rays: binaries

\section{Introduction}

Galactic Black Hole X-ray Binaries (hereafter BH XrBs) are binary systems that were first detected as X-ray sources. They harbor a massive compact object as a primary star, being therefore a black hole candidate, accreting matter from the companion. The X-ray emission probes the inner regions around the compact object and is interpreted as a signature of accretion (Tanaka \& Lewin 1995; McClintock \& Remillard 2003, hereafter McCR03, and references therein). There is now growing evidence that these objects also display ejection signatures: radio emission is commonly interpreted as the presence of compact steady jets or the sporadic ejection events also seen in infrared and X-ray bands (Fender \& Belloni 2004; Buxton \& Bailyn 2004). In fact, the correlation between radio luminosity (ejection) and X-ray luminosity (accretion) found in Active Galactic Nuclei (AGN) seems to be also consistent with XrBs (Robertson \& Leiter 2004; Falcke et al. 2004; Gallo et al. 2003;
Choudhury et al. 2003; Corbel et al. 2003). The similarity of the first detected galactic jet in 1E1740 with extragalactic jets gave birth to the name "microquasar" (Mirabel \& Rodriguez 1998).

What is so dramatic about microquasars is their multiple manifestations through very different spectral states. They spend most of their time in the Quiescent state which is characterized by a very low accretion rate $\left(\dot{m}=\dot{M}_{\mathrm{a}} c^{2} / L_{\text {Edd }}\right.$ as low as $\sim 10^{-9}$ ). The multiwavelength spectral energy distributions are thus very scarse but generally show a hard X-ray $(2-10 \mathrm{keV})$ spectrum with a power law photon index $\Gamma=$ 1.5-2.1 and an optical/UV continuum reminiscent of a $\sim 10^{4} \mathrm{~K}$ disc blackbody with strong emission lines (McCR03).

Occasionally, microquasars enter in outburst, resulting from a dramatic increase of their accretion rate. During these outbursts, microquasars show different canonical states, like the well known Hard and Soft states. The hard state is characterized by a spectrum dominated above $2 \mathrm{keV}$ by a hard 
power-law component $(\Gamma \sim 1.5)$ with a cut-off around $100 \mathrm{keV}$ (e.g. Grove et al. 1998; Zdziarski \& Gierlinski 2003, and references therein), and with a soft X-ray excess below $2 \mathrm{keV}$ interpreted as the presence of a cool $(\sim 0.01-0.2 \mathrm{keV})$ accretion disc. Strong radio emission is observed during this state and some VLBI images directly revealed spatially resolved structures. These are interpreted as non or only mildly relativistic (bulk Lorentz factor $\Gamma_{\mathrm{b}}<2$ ) steady jets (e.g. Stirling et al. 2001; Dhawan et al. 2000). On the contrary, the soft state is dominated by a thermal blackbody-like component, typical of a standard accretion disc of temperature ranging from 0.7 to $1.5 \mathrm{keV}$ (consistent with an inner disc radius $r_{\text {in }} \sim 10 r_{\mathrm{g}}=$ $G M / c^{2}$ ). A faint power-law component may still be present but with a steep photon index $\Gamma=2.1-4.8$ (McCR03). This state is also devoid of any radio emission which is interpreted as the absence of jet (e.g. Fender et al. 1999; Corbel et al. 2000). Hard and soft state span a relatively large range in luminosity, i.e. from $10^{-2}$ to $1 L_{\text {Edd }}$.

Microquasars can also be observed in Intermediate states, generally during transitions between the hard and soft states. Intermediate states present relatively complex spectral and timing behaviors. Detailed studies have been done in the recent literature (see e.g. Fender et al. 2004; Belloni et al. 2005) and reveal a clear evolution with time between hard, variable (in $\mathrm{X}$-ray) and radio-loud systems to softer, less variable and more radio-quiet ones. The transitions between the hard and soft "flavors", which seems to correspond also to a transition between jet-producing and jet-free states, can be relatively abrupt especially at high luminosity level where they are apparently coincident with strong radio outbursts (Fender \& Belloni 2004). These non steady ejection events display apparent superluminal velocities, indicative of a highly relativistic plasma (Mirabel \& Rodríguez 1999; Dhawan et al. 2000). These different observational characteristics lead to the definition of the so-called "jet line" by Fender \& Belloni (2004), that separates hard/jet dominated states to soft/jet-free ones in hardness-intensity diagrams.

These various spectral states obviously carry a huge amount of information about the physical processes behind accretion and ejection. Moreover, since it is believed that microquasars are a scaled down version of AGN, understanding the various states (along with their transitions) in XrBs will certainly provide insights on the observed differences between AGN (radio loud/radio quiet, FRI/FRII, blazars...).

The puzzle of the existence of these different spectral states is enhanced by the fact that each state must correspond to a dynamically steady state. Indeed, each state lasts a much longer time than the inferred dynamical time scale. Let us consider the case of GRS1915+105, which is the $\mathrm{BH} \mathrm{XrB}$ with the most rapid time scales. It shows states lasting $\sim 10^{3} \mathrm{~s}$ with rising and decay times of the order of one second, whereas the Keplerian orbit time scale is several milliseconds at the inner radius (Belloni et al. 1997). One must therefore explain why a system switches from one stationary state to another one. But before that, one must first identify the relevant underlying dynamics describing each state. Up to now, these canonical spectral states are still not fully understood, let alone the transitions between them.
The most common paradigm used to interpret observations is based on the low radiative efficiency of the ADAF model (Esin et al. 1997, and references therein). Within this framework, the highest energy component is due to the inner thick, low radiative disc whereas an outer standard disc is responsible for the UV-soft $\mathrm{X}$ ray emission. By varying the transition radius $r_{t r}$ between these two discs (as a function of $\dot{m}$ ) one gets reasonable fits to the spectral energy distributions (hereafter SEDs, e.g. Narayan et al. 1996; Hameury et al. 1997; Esin et al. 1998, 2001). In the ADAF paradigm, the accretion power below the transition radius is essentially stored as thermal energy of protons and eventually advected below the black hole event horizon. Remarkably, energetic ejection events appear to be always associated with hard and intermediate states and are quenched during the soft phase (McCR03; and references therein). This has led several authors to propose that ADAFs could indeed drive outflows or its ADIOS extension (Blandford \& Begelman 1999, 2004). However, it must be realized that there is only one source of energy, namely the release of gravitational energy through accretion. Hence, whenever a disc is capable of driving jets, these will carry away a fraction of the released accretion energy. As a consequence, the disc luminosity will be quenched. Observations tell us something consistent with this very simple and unavoidable argument: whenever a steady jet is formed the disc may observationally disappear. This does not mean that the disc is really disappearing (i.e., inner region being depleted of its mass), only that we do not see it anymore. Discs that drive jets are indeed radiating only a small fraction of the accretion power released as shown in another class of accretion solution, namely Magnetised Accretion-Ejection Structures (hereafter MAES, see e.g. Ferreira \& Pelletier 1993, 1995; and Ferreira 2002, for a review). In this flow, a large scale magnetic field is threading the disc, exerts a torque leading to accretion and allows the production of self-confined jets. A logical consequence is that whenever a jet is formed, the ADAF hypothesis is no more useful to explain the low radiative efficiency of the accretion flow.

The goal of this paper is to provide an alternative view explaining the canonical spectral states observed in $\mathrm{BH} \mathrm{XrBs}$ based on MAES. We do not intend to address the crucial issue of the transitions between these states, neither time scales involved nor possible hysteresis effects. This is delayed for future work. In this paper, we only expose the global physical picture and show that this framework is rich enough to explain all known spectral components. In a forthcoming paper, we will provide calculations of SEDs and compare them to observations.

The paper is then organized as follows. Section 2 describes in some detail the four physical components present within our paradigm, their dynamical properties as well as radiative processes governing their emission. The canonical spectral states of $\mathrm{BH} \mathrm{XrBs}$ are then interpreted within this framework in Sect. 3. Section 4 is devoted to a discussion of some time scales and timing properties of our model that could be related to some observed features. Section 5 highlights questions opened by our framework as well as future developments. 


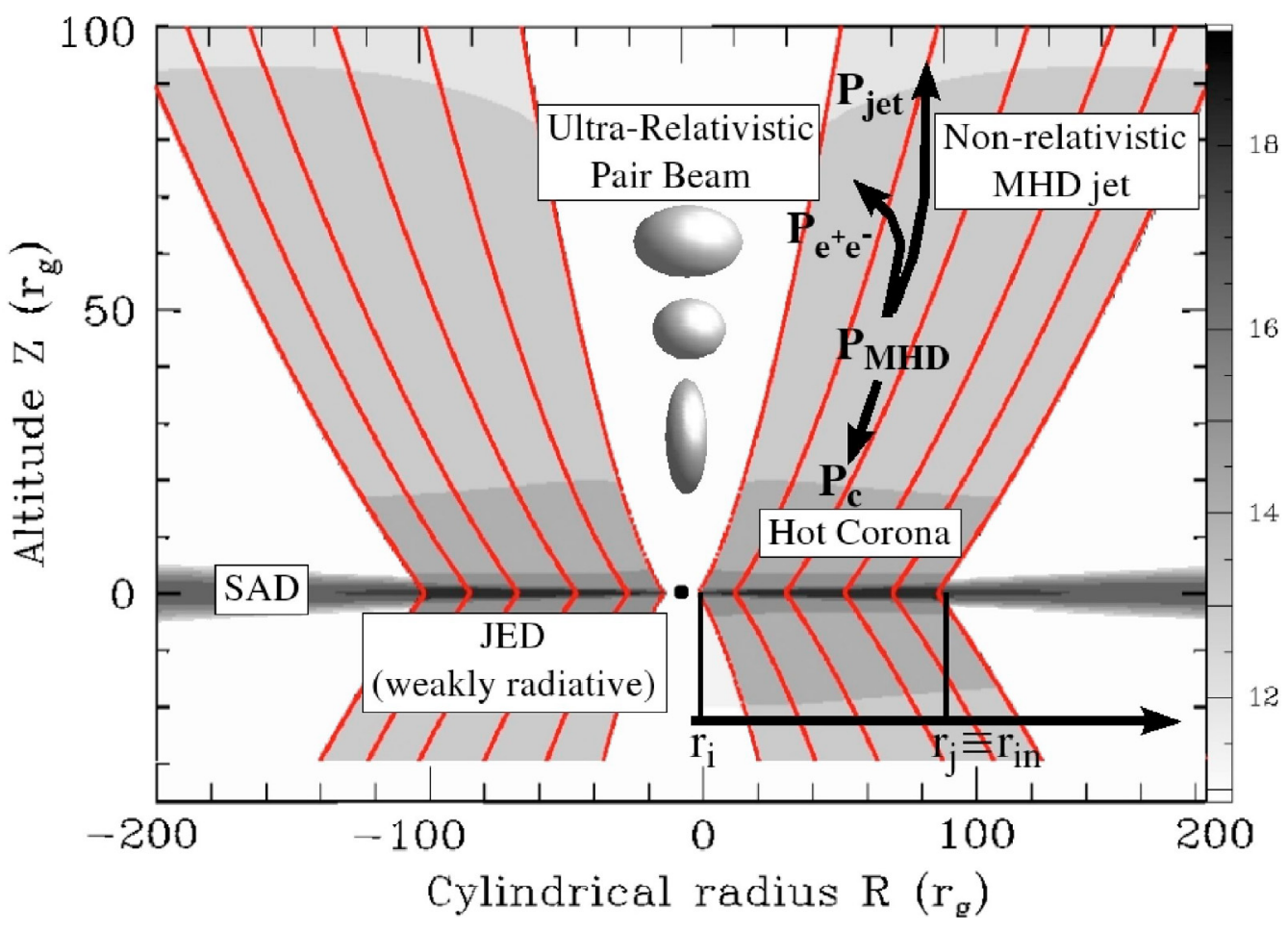

Fig. 1. A Standard Accretion Disc (SAD) is established down to a radius $r_{J}$ which marks the transition towards a low radiative Jet Emitting Disc (JED), settled down to the last stable orbit. The JED is driving a mildly relativistic, self-collimated electron-proton jet which, when suitable conditions are met, is confining an inner ultra-relativistic electron-positron beam. The MHD power $P_{\text {MHD }}$ flowing from the JED acts as a reservoir for (1) heating the jet basis (radiating as a moving thermal corona with power $\left.P_{\mathrm{c}}\right)$; $(2)$ heating the inner pair beam $\left(P_{\left.\mathrm{e}^{+} \mathrm{e}^{-}\right)}\right.$ and (3) driving the compact jet $\left(P_{\text {jet }}\right)$. Field lines are drawn in solid lines and the number density is shown in greyscale $\left(\log _{10} n / \mathrm{m}^{-3}\right)$. The MAES solution (JED and MHD jet) was computed with $\xi=0.01, \varepsilon=0.01$ and with $m=10$ and $\dot{m}\left(r_{J}\right)=0.01$ (see text).

\section{A novel framework for $\mathrm{BH} \mathrm{XrBs}$}

\subsection{General picture}

We assume that the central regions of $\mathrm{BH} \mathrm{XrB}$ are composed of four distinct flows: two discs, one outer "standard" accretion disc (hereafter SAD) and one inner jet emitting disc (hereafter JED), and two jets, a non-relativistic, self-confined electronproton MHD jet and, when adequate conditions for pair creation are fulfilled, a ultra-relativistic electron-positron beam. A sketch of our model is shown in Fig. 1 while the four dynamical components are discussed separately below. This is an extended version of the "two-flow" model early proposed for AGN and quasars (Pelletier et al. 1988; Sol et al. 1989; Pelletier \& Roland 1989; Henri \& Pelletier 1991; Pelletier \& Sol 1992) to explain the highly relativistic phenomena such as superluminal motions observed in these sources. This model provides a promising framework to explain the canonical spectral states of BH XrBs mainly by varying the transition radius $r_{J}$ between the SAD and the JED. This statement is not new and has already been proposed in the past by different authors (e.g. Esin et al. 1997; Belloni et al. 1997; Livio et al. 2003; King et al. 2004) but our model distinguish itself from the others by the consistency of its disc-jet structure and by the introduction of a new physical component, the ultra-relativistic electron-positron beam, that appears during strong outbursts.

We believe that jets from $\mathrm{BH}$ XrBs are self-collimated because they follow the same accretion-ejection correlation as in AGN (Corbel et al. 2003; Fender et al. 2003; Merloni et al. 2003). This therefore implies the presence of a large scale vertical field anchored somewhere in the accretion disc. We think it is unlikely that such a field has a patchy distribution on the disc. Indeed, once a jet is launched, it exerts a torque on the underlying disc with a corresponding vertical flux of angular momentum and energy. This torque writes $-J_{r} B_{z} \sim B_{\phi}^{+} B_{z} / \mu_{0} h<0$ where $B_{\phi}^{+}$is the toroidal field at the disc surface. This shows that the product $J_{r} B_{z}$ must remain positive over the whole region driving jets. Such a condition is unlikely to be met with a vertical field changing its polarity from one zone to another because the currents (both radial and azimuthal) induced inside the disc will tend to cancel each other. The most efficient way to launch jets from an accretion disc is probably from a radial extension (the JED) with a large scale $B_{z}$ of the same polarity.

This vertical field is therefore an unavoidable ingredient to produce self-collimated jets. So, where does this field come from? A first possible origin is in-situ generation of magnetic fields by dynamo. The huge difficulty is to provide an ordered large scale vertical field out of turbulent small scale seed fields. Opening of magnetic loops by the disc differential rotation might provide a mechanism (Romanova et al. 1998). The second origin is advection of magnetic field by the accreting material ${ }^{1}$. We therefore assume that the whole accretion disc is pervaded by a large scale magnetic field $B_{z}$.

1 Note also that a fraction of the open poloidal flux initially tied to the primary's progenitor could remain trapped by the accretion flow. 
The presence of a large scale vertical field threading the disc is however not sufficient to drive super-Alfvénic jets. This field must be close to equipartition as shown by Ferreira \& Pelletier (1995) and Ferreira (1997). The reason is twofold. On one hand, the magnetic field is vertically pinching the accretion disc so that a (quasi) vertical equilibrium is obtained only thanks to the gas and radiation pressure support. As a consequence, the field cannot be too strong. But on the other hand, the field must be strong enough to accelerate efficiently the plasma right at the disc surface (so that the slow-magnetosonic point is crossed smoothly). These two constraints can only be met with fields close to equipartition.

An important local parameter is therefore the disc magnetization $\mu=B_{z}^{2} /\left(\mu_{0} P_{\text {tot }}\right)$ where $P_{\text {tot }}$ includes the plasma and radiation pressures. In our picture, a SAD is established down to a radius $r_{J}$ where $\mu$ becomes of order unity. Inside this radius, a JED with $\mu \sim 1$ is settled. At any given time, the exact value of $r_{J}$ depends on highly non-linear processes such as the interplay between the amount of new large scale magnetic field carried in by the accreting plasma (e.g. coming from the secondary) and turbulent magnetic diffusivity redistributing the magnetic flux already present. These processes are far to be understood. For the sake of simplicity, we will treat in the following $r_{J}$ as a free parameter that may vary with time (see Sect. 3).

\subsection{The outer $S A D$}

Accretion requires the presence of a negative torque extracting angular momentum. In a SAD this torque is assumed to be of turbulent origin and provides an outward transport of angular momentum in the radial direction. It has been modeled as an "anomalous" viscous torque of amplitude $\sim-\alpha_{\mathrm{v}} P_{\text {tot }} / r$, where $\alpha_{\mathrm{v}}$ is a small parameter (Shakura \& Sunyaev 1973). The origin of this turbulence is now commonly believed to arise from the magneto-rotational instability or MRI (Balbus \& Hawley 1991). The MRI requires the presence of a weak magnetic field $(\mu<1)$ and is quenched when the field is close to equipartition. We make the conjecture that a SAD no longer exists once $\mu$ reaches unity. We show below that this is very likely to occur in the innermost regions.

The radial distribution $B_{z}(r)$ is provided by the induction equation which describes the interplay between advection and diffusion. If we assume that, in steady state, the poloidal field is mostly vertical (no significant bending within the SAD) then this equation writes

$$
v_{\mathrm{m}} \frac{\partial B_{z}}{\partial r} \simeq u_{r} B_{z}
$$

where $v_{\mathrm{m}}$ is the turbulent magnetic diffusivity. This equation has the obvious exact solution

$$
B_{z} \propto r^{-\mathcal{R}_{\mathrm{m}}}
$$

where $\mathcal{R}_{\mathrm{m}}=-r u_{r} / v_{\mathrm{m}}$ is the (effective) magnetic Reynolds number. In a turbulent disc one usually assumes that all anomalous transport coefficients are of the same order so that $v_{\mathrm{m}} \simeq v_{\mathrm{v}}$, $v_{\mathrm{v}}$ being the turbulent viscosity. Since the (effective) Reynolds number $\mathcal{R}_{\mathrm{e}}=-r u_{r} / v_{\mathrm{v}}=3 / 2$ in a SAD, one gets that any vertical magnetic field is naturally increasing towards the center. Now in a SAD of vertical scale height $h(r) \propto r^{\delta}$, the total pressure $P_{\mathrm{tot}}=\rho \Omega_{\mathrm{k}}^{2} h^{2}\left(\Omega_{\mathrm{k}}\right.$ is the Keplerian rotation rate) scales as

$P_{\text {tot }}=\frac{\dot{M}_{\mathrm{a}} \Omega_{\mathrm{k}}^{2} h}{6 \pi v_{\mathrm{v}}} \propto r^{-3 / 2-\delta}$

where $\dot{M}_{\mathrm{a}}$ is the (constant) disc accretion rate. Using Eq. (2) we get

$\mu \propto r^{-\epsilon}$ with $\epsilon=2 \mathcal{R}_{\mathrm{m}}-\delta-3 / 2$.

In a $\operatorname{SAD} \mathcal{R}_{\mathrm{m}} \simeq 3 / 2$ and $\delta$ is always close to unity (apart from the unstable radiation pressure dominated zone where $\delta=0$ ). Of course, the real value of $\epsilon$ critically depends on the magnetic Prandtl number $\left(\mathcal{P}_{\mathrm{m}}=v_{\mathrm{v}} / v_{\mathrm{m}}\right)$ but this result suggests that one may reasonably expect $\mu$ to increase towards the center. Whenever a $\mathrm{BH} \mathrm{XrB}$ reaches $\mu \simeq 1$ at a radius $r_{J}>r_{i}, r_{i}$ being the last marginally stable orbit, the accretion flow changes its nature to a JED.

To summarize, the accretion flow at $r>r_{J}$ is a SAD with $\mu \ll 1$ fueled by the companion's mass flux and driving no outflow (constant accretion rate $\dot{M}_{\mathrm{a}}$ ). The global energy budget is $P_{\mathrm{acc}, \mathrm{SAD}}=2 P_{\mathrm{rad}, \mathrm{SAD}}$ where

$$
P_{\mathrm{acc}, \mathrm{SAD}} \simeq \frac{G M \dot{M}_{\mathrm{a}}}{2 r_{J}}
$$

and $P_{\text {rad,SAD }}$ is the disc luminosity (from one surface only). Its emission has the characteristic multi-blackbody shape produced by a radial temperature distribution $T_{\text {eff }}(r) \propto r^{-3 / 4}$. The spectrum is therefore dominated by the hottest inner parts at $r_{J}$.

\subsection{The inner JED}

This inner region with $\mu \sim 1$ is fueled by the SAD at a rate $\dot{M}_{\mathrm{a}, J}=\dot{M}_{\mathrm{a}}\left(r_{J}\right)$. Since it undergoes mass loss, the JED accretion rate is written as

$\dot{M}_{\mathrm{a}}(r)=\dot{M}_{\mathrm{a}, J}\left(\frac{r}{r_{J}}\right)^{\xi}$

where $\xi$ measures the local ejection efficiency (Ferreira \& Pelletier 1993). The global energy budget in the JED is $P_{\text {acc,JED }}=2 P_{\text {rad,JED }}+2 P_{\text {MHD }}$ where $P_{\text {MHD }}$ is the MHD Poynting flux feeding a jet, whereas the liberated accretion power writes

$P_{\mathrm{acc}, \mathrm{JED}} \simeq \frac{G M \dot{M}_{\mathrm{a}, J}}{2 r_{i}}\left[\left(\frac{r_{i}}{r_{J}}\right)^{\xi}-\frac{r_{i}}{r_{J}}\right]$.

The dynamical properties of a JED have been extensively studied in a series of papers (see Ferreira 2002, and references therein). We will here only briefly recall the main properties and refer the interested reader to specific papers. In this dynamical structure accretion and ejection are interdependent: jets carry away the exact amount of angular momentum allowing the disc material to accrete. The ratio at the disc midplane of the jet torque to the turbulent "viscous" torque is

$$
\Lambda \sim \frac{B_{\phi}^{+} B_{z} / \mu_{\mathrm{o}} h}{\alpha_{\mathrm{v}} P_{\mathrm{tot}} / r} \sim \frac{B_{\phi}^{+} B_{z}}{\mu_{\mathrm{o}} P_{\mathrm{tot}}} \frac{r}{\alpha_{\mathrm{v}} h} .
$$


It is straightforward to see that the necessary condition to drive jets (fields close to equipartition) from Keplerian discs leads to a dominant jet torque. In fact, it has been shown that steadystate ejection requires $\Lambda \sim r / h \gg 1$ (Ferreira 1997; Casse \& Ferreira 2000a).

This dynamical property has a tremendous implication on the JED emissivity. The JED luminosity comes from the accretion power dissipated within the disc by turbulence and transported away by photons, so $2 P_{\text {rad,JED }}=P_{\text {diss }}$. This dissipated power is very difficult to estimate with precision because it requires a thorough description of the turbulence itself. Thus, one usually uses crude estimates based on "anomalous" turbulent magnetic resistivity $\eta_{\mathrm{m}}$ (Joule heating) and viscosity $\eta_{\mathrm{v}}$ (viscous heating). This translates into $P_{\text {diss }}=P_{\text {Joule }}+P_{\text {visc }}=$ $\int \eta_{\mathrm{m}} J^{2} \mathrm{~d} V+\int \eta_{\mathrm{v}}(r \partial \Omega / \partial r)^{2} \mathrm{~d} V$ where integration is made over the whole volume occupied by the JED. The importance of local "viscous" dissipation with respect to the MHD Poynting flux leaving the disc is approximately given by

$$
\frac{P_{\mathrm{visc}}}{2 P_{\mathrm{MHD}}} \sim \frac{1}{\Lambda}
$$

which is much larger than unity: turbulent "viscosity" provides negligible dissipation in a JED. Joule heating arises from the dissipation of toroidal and radial currents which are comparable ${ }^{2}$. One therefore gets $\eta_{\mathrm{m}} J^{2} \sim \nu_{\mathrm{m}} B_{z}^{2} / \mu_{\mathrm{o}} h^{2} \sim v_{\mathrm{v}} \rho \Omega^{2} \sim$ $\eta_{\mathrm{v}}(r \partial \Omega / \partial r)^{2}$, for equipartition fields, isotropic magnetic resistivity $\eta_{\mathrm{m}}=\mu_{\mathrm{o}} \nu_{\mathrm{m}}$ and a turbulent magnetic Prandtl number of order unity. This leads to

$\frac{P_{\mathrm{Joule}}}{2 P_{\mathrm{MHD}}} \sim \frac{1}{\Lambda}$

namely a negligible effective Joule heating. Thus, the total luminosity $2 P_{\text {rad,JED }}$ of the JED is only a fraction $1 /(1+\Lambda)$ of the accretion disc liberated power $P_{\text {acc,JED}}$.

To summarize, under quite general conditions on the turbulence within magnetized discs, most of the available accretion energy is powering the outflowing plasma (Ferreira \& Pelletier 1993, 1995). This is in strong contrast with ADAFs where the accretion power is stored as heat advected by the accreting plasma. In this case, low luminosity discs can be obtained as long as the central object possesses an event horizon. However, the power to magnetically drive jets is also missing. In the case of MAES, the JED is weakly dissipative while powerful jets are being produced regardless of the nature of the central object.

Complete calculations of MAES showed that isothermal or adiabatic super-Alfvénic jets from Keplerian accretion discs were possible only when a tiny fraction of the accreted mass is locally ejected. This translates into a small ejection efficiency, typically $\xi \sim 0.01$ (Ferreira 1997; Casse \& Ferreira 2000a). When some heat deposition occurs at the JED upper layers a typical value of $\xi \sim 0.1$ becomes possible, even up to 0.5 but never reaching unity, in agreement with Eq. (7) (Casse \& Ferreira 2000b). This is a much lower mass loss than that assumed in ADIOS models (Blandford \& Begelman 1999).

\footnotetext{
2 Full computations of MAES show that the three magnetic field components are comparable at the disc surface, namely $B_{\phi}^{+} \sim B_{r}^{+} \sim B_{z}$ (Ferreira \& Pelletier 1995; Ferreira 1997).
}

The fact that the jet torque largely dominates the turbulent torque provides another striking difference between the internal structures of SADs and JEDs. Indeed, the angular momentum conservation provides a sonic Mach number measured at the disc midplane

$m_{\mathrm{s}}=-\frac{u_{r}}{C_{\mathrm{s}}}=\alpha_{\mathrm{v}} \varepsilon(1+\Lambda)$

where $C_{\mathrm{s}}=\Omega_{\mathrm{k}} h$ is the sound speed. Thus, a SAD displays $m_{\mathrm{S}}=\alpha_{\mathrm{v}} \varepsilon \ll 1$ whereas a JED has a much higher accretion velocity, namely $m_{\mathrm{s}} \simeq 1$ (Ferreira \& Pelletier 1995; Ferreira 1997). This has two major consequences. First, a JED is much less dense than a $\mathrm{SAD}^{3}$. Second, there is a stronger bending of the poloidal field lines. Indeed, in spite of the same turbulent magnetic diffusivity $\left(v_{\mathrm{m}} \sim v_{\mathrm{v}}\right)$, the larger accretion velocity $u_{r}$ leads to an effective magnetic Reynolds number $\mathcal{R}_{\mathrm{m}} \sim \varepsilon^{-1}$ where $\varepsilon=h / r$ is the disc aspect ratio (Ferreira \& Pelletier 1995). This translates into a field at the disc surface verifying $B_{r}^{+} / B_{z} \sim \mathcal{R}_{\mathrm{m}} \varepsilon \gtrsim 1$, as required to magnetically launch cold jets.

Mass conservation in the JED writes

$$
\begin{aligned}
n & =\frac{\dot{M}_{\mathrm{a}}(r)}{4 \pi m_{\mathrm{p}} \Omega_{\mathrm{k}} r^{3}} m_{\mathrm{s}}^{-1} \varepsilon^{-2} \\
& \simeq 10^{25} \varepsilon^{-2} \dot{m} m^{-1} R^{\xi-\frac{3}{2}} \mathrm{~m}^{-3}
\end{aligned}
$$

where $m=M / M_{\odot}, R=r / r_{\mathrm{g}}\left(r_{\mathrm{g}}=G M / c^{2}\right)$ and $\dot{m}=$ $\dot{M}_{\mathrm{a}, J} c^{2} / L_{\mathrm{Edd}}$. This density requires a magnetic field

$$
\begin{aligned}
B_{z} & =\left(\frac{\mu}{m_{\mathrm{s}}}\right)^{1 / 2}\left(\frac{\mu_{\mathrm{o}} \dot{M}_{\mathrm{a}}(r) \Omega_{\mathrm{k}}}{4 \pi r}\right)^{1 / 2} \\
& \simeq 4.4 \times 10^{8} \dot{m}^{1 / 2} m^{-1 / 2} R^{\frac{\xi}{2}-\frac{5}{4}} \mathrm{G} .
\end{aligned}
$$

For illustration, we provide below the case of an optically thick, Thomson dominated and gas pressure supported JED. In this region, the disc aspect ratio is

$\varepsilon=h / r=2.6 \times 10^{-3} \dot{m}^{1 / 4} m^{-1 / 8} R^{\frac{\xi}{4}+\frac{1}{16}}$

which allows to precisely specify the above quantities. Moreover, the ratio of radiation to gas pressure, Thomson opacity, effective and central temperatures are

$$
\begin{aligned}
\frac{P_{\mathrm{rad}}}{P_{\mathrm{gas}}} & =0.3 \dot{m} R^{\xi-1} \\
\tau_{\mathrm{T}} & \simeq n \sigma_{\mathrm{T}} h=3.8 \times 10^{2} \dot{m}^{3 / 4} m^{1 / 8} R^{\frac{3 \xi}{4}-\frac{9}{16}} \\
T_{\mathrm{eff}} & \simeq 876 \dot{m}^{5 / 16} m^{-9 / 32} R^{\frac{5 \xi}{16}-\frac{47}{64}} \mathrm{eV} \\
T_{\mathrm{o}} & =3.2 \dot{m}^{1 / 2} m^{-1 / 4} R^{\frac{\xi}{2}-\frac{7}{8}} \mathrm{keV} .
\end{aligned}
$$

The spectrum emitted by an optically thick JED is a multiblackbody but with a temperature drastically reduced from that of a SAD. As a consequence, the flux emitted by the JED is expected to be unobservable with respect to that of the outer SAD. In practice, this mostly depends on the radial extension of the JED. Indeed, the flux emitted by the surrounding

\footnotetext{
3 It has been recently showed that a previously claimed instability of accretion-ejection structures does not apply to this type of solution (see Königl 2004, and references therein).
} 
SAD scales as $T_{\mathrm{eff}, \mathrm{SAD}}^{4} r^{2}$ measured at $r_{J}$ whereas the flux emitted by the JED is dominated by $T_{\mathrm{effJ} J \mathrm{JE}}^{4} r^{2}$ measured at $r_{i}$. One therefore gets that the ratio of the JED to the SAD flux scales as $\left(r_{J} / r_{i}\right) /(1+\Lambda) \sim \varepsilon r_{J} / r_{i}$ which is much smaller than unity for reasonable values of $r_{J}$. Thus, the values of the "disc inner radius" $\left(r_{\text {in }}\right)$ and "disc accretion rate" observationally determined from spectral fits must be understood here as values at the transition radius, namely $r_{\text {in }} \equiv r_{J}$ and $\dot{m} \equiv \dot{m}\left(r_{J}\right)$ : the optically thick JED is spectrally hardly visible.

\subsection{Non-relativistic electron-proton jets from JEDs}

The ejection to accretion rate ratio in a JED writes $2 \dot{M}_{\text {jet }} / \dot{M}_{\mathrm{a}, J} \simeq \xi \ln \left(r_{J} / r_{i}\right)$. In principle, the ejection efficiency $\xi$ can be observationally deduced from the terminal jet speed. Indeed, the maximum velocity reachable along a magnetic surface anchored on a radius $r_{\mathrm{o}}$ (between $r_{i}$ and $r_{J}$ ) is $u_{\infty} \simeq$ $\xi^{-1 / 2} \sqrt{G M / r_{\mathrm{o}}}$ in the non-relativistic limit (see Ferreira 1997, for relativistic estimates). Although a large power is provided to the ejected mass (mainly electrons and protons), the mass loss $(\xi)$ is never low enough to allow for speeds significantly relativistic required by superluminal motions: MHD jets from accretion discs are basically non or only mildly relativistic with $u_{\infty} \sim 0.1-0.8 c$ (Ferreira 1997). This is basically the reason why they can be efficiently self-confined by the magnetic hoop stress. Indeed, in relativistic flows the electric field grows so much that it counteracts the confining effect due to the toroidal field. This dramatically reduces the self-collimation property of jets (Bogovalov \& Tsinganos 2001; Bogovalov 2001; Pelletier 2004).

Calculations of jets crossing the MHD critical points have been undergone under the self-similar ansazt (Casse \& Ferreira 2000b; Ferreira \& Casse 2004). In these calculations, the emission of the MHD jet has been neglected and all the available power is converted into ordered jet kinetic energy. However, a fraction of this power is always converted into heat and particle acceleration, leading to emission. In our case, jets from MAES have two distinct spectral components and the resultant SED may therefore be quite intricate. Producing a global SED is out of the scope of the present paper. It requires to fix several parameters, which is legitimate only by object fitting. This is postponed for future work.

\subsubsection{A non-thermal extended jet emission}

We expect a small fraction of the jet power $P_{\text {jet }}$ to be converted into particles, through first and/or second order Fermi acceleration, populating the MHD jet with supra-thermal particles. These particles are responsible for the bulk emission of the MHD jet. This is similar to models of jet emission already proposed in the literature (Falcke \& Biermann 1995; Vadawale et al. 2001; Markoff et al. 2001, 2003; Markoff 2004; Falcke et al. 2004). In these models, the jet is assumed to be radiating self-absorbed synchrotron emission in the radio band becoming then optically thin in the IR-Optical bands and providing a contribution up to the $\mathrm{X} / \gamma$-rays. A flat or even inverted spectrum index in the radio band is quite easily achieved by self-collimated jets for reasonable values of the parameters (e.g. the exponent $p$ of the power law particle distribution).

Note that the MHD jet due to the MAES yields $B \propto$ $m^{-1 / 2} \dot{m}^{1 / 2}$. In the framework of Heinz \& Sunyaev (2003), this implies that such a jet would provide correlated radio and X-ray emissions close to the observed law, namely $F_{R} \propto F_{\mathrm{X}}^{0.7}$ Gallo et al. (2003); Corbel et al. (2003). However, the spectrum index in the X-ray band would not be steep enough, even taking into account the cooling of the particles. Moreover, the fundamental plane of BH activity of Merloni et al. (2003), namely the correlation between mass, radio and X-ray fluxes, cannot be explained by such synchrotron jets (Heinz 2004). Following these authors, we conclude that there must be another significant contribution to the X-ray emission in the Low/hard state. The same conclusion was independently reached by Rodriguez et al. (2004) who suggested that, to explain the energy dependence of the quasi-periodic oscillation (QPO) amplitude in GRS $1915+105$, the high energy spectrum of the source must be the sum of different emission processes. Another argument comes from the study of the overall spectral energy distributions. At least in some objects, the extrapolation of the X-ray power-law spectrum towards the optical and infrared bands is above the observed fluxes, which shows that hard X-rays cannot be direct synchrotron radiation from the jet (Kalemci et al. 2005).

Moreover, $\mathrm{BH} \mathrm{XrBs}$ in the hard state generally exhibit a spectrum with a high energy cut-off around $100 \mathrm{keV}$ (e.g. Grove et al. 1998; Zdziarski \& Gierlinski 2003, and references therein). While naturally obtained if the emission is thermal (i.e. a comptonized corona), non-thermal emission requires a fine tuning of the parameters that we found questionable. However, our framework naturally provides another contribution to the high energy emission as long as a JED is present.

\subsubsection{A thermal jet basis}

Jet production relies on a large scale magnetic field anchored on the disc as much as on MHD turbulence triggered (and sustained) within it. This implies that small scale magnetic fields are sheared by the disc differential rotation, leading to violent release of magnetic energy at the disc surface and related turbulent heat fluxes (e.g. Galeev et al. 1979; Heyvaerts \& Priest 1989; Stone et al. 1996; Merloni \& Fabian 2002). The energy released is actually tapping the MHD Poynting flux flowing from the disc surface. We can safely assume that a fraction $f$ of it would be deposited at the jet basis, with a total power $P_{\mathrm{c}}=f P_{\mathrm{MHD}}$. The dominant cooling term in this optically thin medium is probably comptonization of soft photons emitted by the outer SAD (with a small contribution from the underlying JED). These are circumstances allowing a thermal plasma to reach a temperature as high as $\sim 100 \mathrm{keV}$, (Pietrini \& Krolik 1995; Mahadevan 1997; Esin et al. 1997).

The computation of the exact spectral shape produced by this "corona" through thermal comptonization requires sophisticated computations (e.g. Haardt 1993; Poutanen \& Svensson 1996) which are out of the scope of this paper. Instead, a cut-off power law shape is generally used as zero-order 
approximation. In this case, the high energy cut-off is rougly equal to twice the plasma temperature (see e.g. Petrucci et al. 2000 , for more discussion). The power law photon index can also be approximated by a simple fonction of the Compton amplification factor $A$, which is equal to the ratio of the total luminosity outgoing from the jet basis to the soft luminosity $P_{\text {soft }}$ entering in it (see e.g. Beloborodov 1999; Malzac et al. 2001):

$\Gamma \simeq C(A-1)^{-\eta}$ with $A=1+P_{\mathrm{c}} / P_{\text {soft }}$

where $C$ and $\eta$ depend on the geometry of the disc-corona configuration.

In the most general case, $P_{\text {soft }}$ should include the SAD and JED emissions but also the reprocessed radiation from the discs (both JED and SAD) that are partly intercepted by the corona. In consequence, the photon index depends implicitly on parameters like $f, \Lambda, r_{J}$ but also on the bulk motion of the corona in a complex manner. This will be precisely discussed in a forthcoming paper where calculations of SEDs will be provided. Simple estimates can be given, however, in the case of large $r_{J}$ and $\Lambda$, since in these conditions the SAD and JED emissions become negligible compared to the reprocessed one. This situation has been precisely studied by Malzac et al. (2001). The parameters $C$ and $\eta$ of Eq. (19) obtained by these authors are equal to 2.19 and $2 / 15$ respectively. Hard X-rays photon indexes in the range 1.4-2 are easily obtained for different corona velocities and corona aspect ratios (i.e. height/width). These values are in good agreement with what is generally observed in the hard states where we expect large $r_{J}$ (cf. Sect. 3 for a more detailed discussion). We can note also that a decrease of $r_{J}$ will result in a larger $P_{\text {soft }}$, due to the increase of the SAD emission, and thus in a softening of the X-ray spectrum.

\subsection{The inner ultra-relativistic pair beam}

Since the large scale magnetic field driving the self-confined jet is anchored onto the accretion disc which has a non zero inner radius, there is a natural hole on the axis above the central object with no baryonic outflow (this also holds for neutron stars). This hole provides a place for pair production and acceleration with the outer MHD jet acting as a sheath that confines and heats the pair plasma. This is the microquasar version of the "two flow" model that has been successfully applied to the high energy emission of relativistic jets in AGNs (Henri \& Pelletier 1991; Marcowith et al. 1995, 1998; Renaud \& Henri 1998).

The $\mathrm{e}^{+}-\mathrm{e}^{-}$plasma is produced by $\gamma-\gamma$ interaction, the $\gamma$-ray photons being initially produced by a few relativistic particles by Inverse Compton process, either on synchrotron photons (Synchrotron Self Compton or SSC) or on disc photons (External Inverse Compton or EIC). Detailed models for AGNs have shown that all processes can contribute, depending on the physical parameters of the system (magnetic field, disc luminosity, distance). We do not intend to build an explicit Spectral Energy Distribution of the pair plasma here, but we will just discuss the general mechanism of pair beam formation and the relevance of each process in explaining the various non thermal components.

It is well known that above $0.5 \mathrm{MeV}$ photons can annihilate with themselves to produce an electron-positron pair. Usually, pairs are assume to cool once they are formed, producing at turn non thermal radiation. Some of this radiation can be absorbed to produce new pairs, but the overall pair yield never exceeds $10 \%$. A key point of the two-flow model however is that the MHD jet launched from the disc can carry a fair amount of turbulent energy, most probably through its MHD turbulent waves spectrum. A fraction of this power can be transferred to the pairs $\left(P_{\mathrm{e}^{+} \mathrm{e}^{-}} \ll P_{\mathrm{MHD}}\right)$. Thus the freshly created pairs can be continuously reheated, triggering an efficient pair runaway process leading to a dense pair plasma (Henri \& Pelletier 1991).

As we said, reacceleration is balanced by cooling through the combination of synchrotron, SSC and EIC processes. Synchrotron and SSC emission are quasi isotropic in the pair frame, but the external photon field is strongly anisotropic. The pair plasma will then experience a strong bulk acceleration due to the recoil term of EIC, an effect also known as the "Compton Rocket" effect (O'Dell 1981; Renaud \& Henri 1998). As was shown in previous works, this "rocket" effect is the key process to explain relativistic motions (Marcowith et al. 1995; Renaud \& Henri 1998).

At a given distance of the disc, the bulk acceleration saturates at a characteristic Lorentz factor, depending only on the radiation angular distribution. It is defined by the condition that the net radiation flux in the comoving frame (after Lorentz transform) vanishes. It can be shown easily that this characteristic Lorentz factor is approximately $\Gamma_{\mathrm{b}, \mathrm{eq}} \simeq\left(z / r_{i}\right)^{1 / 4}$ on the axis of a standard accretion disc (Renaud \& Henri 1998), as long as the distance $z$ verifies $r_{J} \ll z$ : here the relevant disc inner radius is in fact the transition radius $r_{J}$. Noticeably this value does not depend on the disc luminosity (or accretion rate): it is only dependent on the angular distribution of the intensity, i.e. the radial dependency of the temperature $T \propto r^{-3 / 4}$. The photon field is in fact dominated by photons emitted at $r \sim z$. The modification introduced by the JED in the central region is likely to be immaterial for two reasons. First although the luminosity decreases, the radial dependency remains almost unchanged. Second, as we argue below, the final bulk Lorentz factor depends only on the distance where the plasma decouples from the radiation, and not on the motion close to the core.

A pure pair plasma will thus experience a continuous bulk acceleration, the bulk velocity increasing slowly with the distance. At some point, the radiation field becomes too weak to act efficiently: this happens when the relaxation time towards the equilibrium Lorentz factor becomes larger than the dynamical time $z / c$. At this distance, the acceleration process stops and the plasma decouples from the external radiation field, moving on at a ballistic constant Lorentz factor $\Gamma_{b, \infty}$. The asymptotic Lorentz factor depends essentially on the location of this critical distance.

In the original O'Dell's version of this process, the pair plasma was not supposed to be reheated and this effect has been shown to be rather inefficient, because cooling is always much faster than acceleration (Phinney 1987). In fact, for a cold plasma, the mechanism reduces to the ordinary radiation pressure. Under these conditions, the critical distance is approximately $\ell_{\mathrm{s}}^{4 / 7}$, where $\ell_{\mathrm{s}}=\sigma_{\mathrm{T}} P_{\mathrm{rad}, \mathrm{SAD}} / 4 \pi m_{\mathrm{e}} c^{3} r_{J}$ is the soft photon compactness. For a near Eddington accreting disc, $\ell_{\mathrm{s}} \simeq 10^{3}$ and $\Gamma_{\mathrm{b}, \infty} \sim \ell_{\mathrm{s}}^{1 / 7} \sim 2-3$ (Phinney 1987). Although this is indeed a 
relativistic motion (an apparent superluminal motion is possible), this may not be high enough to account for high values around 5, as observed in microquasars.

In the two flow model however, continuous reheating of the pairs makes the bulk acceleration more efficient, acting thus over a much larger distance: the radiation force is multiplied by $\left\langle\bar{\gamma}^{2}\right\rangle$, where $\bar{\gamma}$ is the random (or relativistic temperature) of the pair plasma, (not to be confused with the bulk Lorentz factor $\Gamma_{b}$ ). Although the equilibrium Lorentz factor at a given distance is unchanged, the critical decoupling distance is much larger. The asymptotic bulk Lorentz factor becomes $\left(\ell_{\mathrm{s}}\left\langle\bar{\gamma}^{2}\right\rangle /\langle\bar{\gamma}\rangle\right)^{1 / 7}$ and values of 5 to 10 can be easily reached in near-Eddington accretion regime around stellar black holes (Renaud \& Henri 1998).

Producing this pair plasma requires thus altogether a strong MHD jet, a radiative non-thermal component extending above the $\mathrm{MeV}$ range and a minimal $\gamma-\gamma$ optical depth, namely $\tau_{\gamma \gamma} \sim$ 1. The non thermal component can indeed be associated with the steep power law observed during the intermediate states, given the fact that it seems to extend to $\mathrm{MeV}$ range without any break (McCR03). It is most probably due to Inverse Compton process on the disc photons. Indeed, the optical depth $\tau_{\gamma \gamma}$ for absorbing photons with energy $E_{\gamma}=\varepsilon m_{\mathrm{e}} c^{2}$ is approximately for a spherical source of radius $R$ filled by soft photons with density $n(\varepsilon)$ by unit reduced energy:

$\tau_{\gamma \gamma}=\frac{1}{\varepsilon} n\left(\frac{1}{\varepsilon}\right) \sigma_{\mathrm{T}} R=\frac{\sigma_{\mathrm{T}} \varepsilon}{4 \pi m_{\mathrm{e}} c^{3} R}\left(v L_{v}\right)_{m_{\mathrm{e}} c^{2} / h \varepsilon}$.

We take a typical soft power-law spectrum $v L_{v}=E L_{E}=$ $L_{0}\left(E / E_{0}\right)^{-\Gamma+2}$ where $\Gamma$ is the soft photon index, typically around 2.5 for luminous intermediate states. Adopting this nominal value, the $\gamma-\gamma$ optical depth becomes a fonction of the energy $E_{\gamma}$ :

$$
\begin{aligned}
\tau_{\gamma \gamma}\left(E_{\gamma}\right)= & 0.7 \times 262^{(2.5-\Gamma)}\left(\frac{L_{0}}{0.1 L_{\mathrm{Edd}}}\right)\left(\frac{R}{30 r_{\mathrm{g}}}\right)^{-1} \\
& \times\left(\frac{E_{0}}{1 \mathrm{keV}}\right)^{\Gamma-2}\left(\frac{E_{\gamma}}{1 \mathrm{MeV}}\right)^{\Gamma-1} .
\end{aligned}
$$

Thus, assumptions that appear quite reasonable for the luminous intermediate state provide good conditions for pair creation.

It is noteworthy that the pair beam is intrinsically highly variable and subject to an intermittent behavior. Indeed, once the pair creation is triggered, a regulation mechanism must occur to avoid infinite power of the pair plasma and limit the pair run-away. This is probably accomplished by the quenching of the turbulence $\left(P_{\mathrm{e}^{+} \mathrm{e}^{-}}\right.$vanishes) when most of its energy is suddenly tapped by the catastrophic number of newly created pairs. These pairs will therefore simply expand freely, confined by the heavier MHD jet. One would then expect a flare in the compact region, followed by the ejection of a superluminal radio component, analoguous to those observed in AGNs (Saugé \& Henri 2005, A\&A, submitted). Such a situation can repeat itself as long as the required physical conditions are met. Alternatively, it may also that the formation of a dense pair beam destroys the surrounding MHD jet, explaining the disappearance of the compact jet after a strong ejection event.

\section{Canonical spectral states of X-ray binaries}

\subsection{The crucial roles of $r_{J}$ and $\dot{m}$}

From Sect. 2, it is clear that the spectral appearance of a $\mathrm{BH} \mathrm{XrB}$ critically depends on the size of the JED relative to the $\mathrm{SAD}$, namely $r_{J}$. As stated before, $r_{J}$ is the radius where the disc magnetization $\mu=B_{z}^{2} /\left(\mu_{\mathrm{o}} P_{\text {tot }}\right)$ becomes of order unity. Thus, $r_{J}$ depends on two quantities $P_{\text {tot }}(r, t)$ and $B_{z}(r, t)$. The total pressure is directly proportional to $\dot{m}$ since $P_{\text {tot }}=\rho \Omega_{\mathrm{k}}^{2} h^{2} \propto$ $\dot{m m^{-1}} r^{-5 / 2}$. As a consequence, any variation of the accretion rate in the outer SAD implies also a change in the amplitude of the total pressure. But we have to assume something about the time evolution of the large scale magnetic field threading the disc. Within our framework, $\dot{m}$ and $B_{z}$ are two quantities that may vary independently with time.

Let us assume that $B_{z}$ does not depend on the disc accretion rate. If, for instance, $\dot{m}$ undergoes a sudden increase (triggered by, e.g. some disc instability at the outer radii Lasota et al. 1996), then there is a corresponding increase of $P_{\text {tot }}$ which is propagating inwards, eventually reaching the inner JED. Since no magnetic flux is being simultaneously added, the region where the vertical magnetic field is close to equipartition shrinks, namely $r_{J}$ decreases. If, on the contrary, the disc accretion rate decreases (without a corresponding decrease of the magnetic flux threading the disc), then the decrease of $P_{\text {tot }}$ requires some diffusion of the vertical magnetic field in the inner regions in order to maintain equipartition, hence the JED expands and $r_{J}$ increases. According to this simplistic argument, one would expect an anti-correlation between $r_{J}$ and $\dot{m}$, namely a larger $\dot{m}$ implies a smaller $r_{J}$ (and vice-versa).

Alternatively, one could also argue that a larger $\dot{m}$ implies more plasma within the disc and that a larger $B_{z}$ would then be locally generated by dynamo. If such a process provides $B_{z} \propto \dot{m}^{1 / 2}$, then $r_{J}$ would always remain unchanged, whatever $\dot{m}$. Another alternative could be advection of the companion's magnetic field along with the flow. Now, because of the stellar dynamo, such a field could also change with a time scale very different from that related to changes in $\dot{m}$. In any case, the amount and polarity advected along strong accretion phases would be an unknown function $B_{z}(\dot{m})$.

The processes governing the amplitude and time scales of these adjustments of $r_{J}$ to a change in $\dot{m}$ are far too complex to be addressed here. They depend on the nature of the magnetic diffusivity within the disc but also on the radial distribution of the vertical magnetic field. We will simply assume in the following that $r_{J}$ and $\dot{m}$ are two independent parameters. In that respect, our view is very different from that of Esin et al. (1997); Mahadevan (1997) who considered only the dependency of $\dot{m}$ to explain the different spectral states of $\mathrm{BH}$ XrBs.

\subsection{The quiescent state}

This state is characterized by a very low accretion rate ( $\dot{m}$ as low as $\sim 10^{-9}$ ) with a hard X-ray component. The ADAF model has been successfully applied to some systems with a large transition radius between the ADAF and the outer standard disc, namely $r_{\text {tr }} \sim 10^{3}-10^{4} r_{\mathrm{g}}$ (e.g. Narayan et al. 1996; 


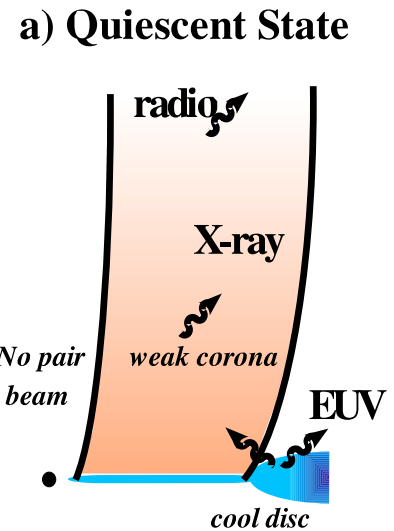

c) Soft State

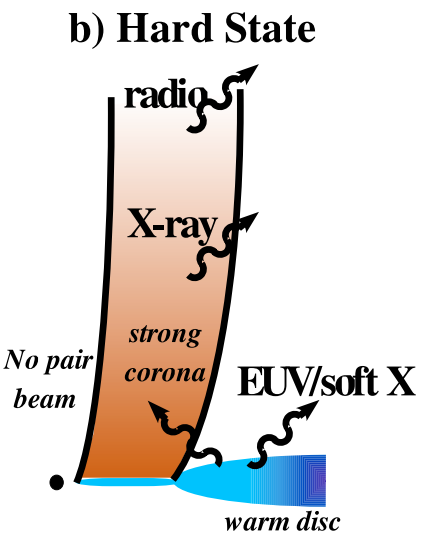

warm disc
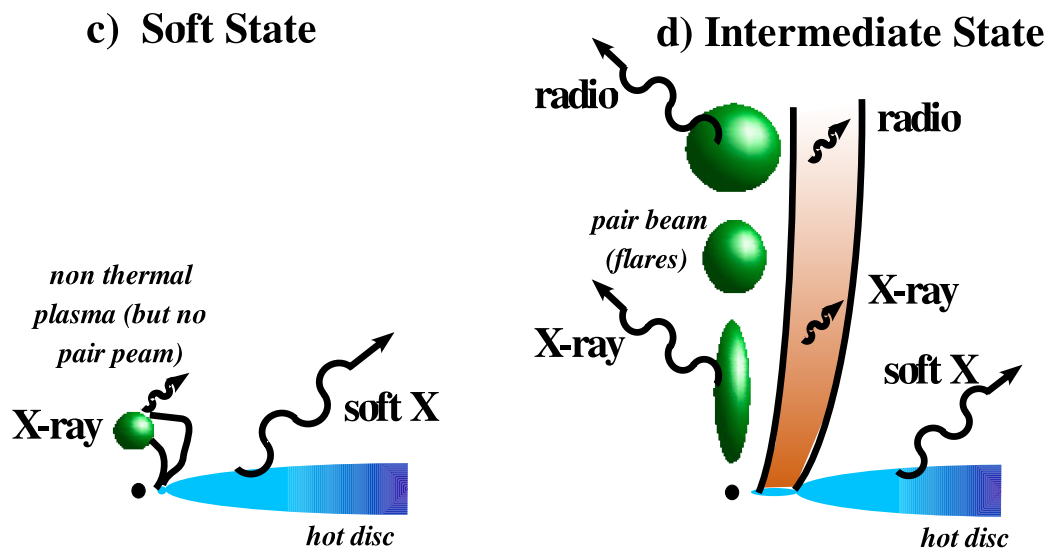

Fig. 2. The canonical spectral states of BH X-ray binaries. a) Quiescent state obtained with a low $\dot{m}$ and a large $r_{J}$ : the Jet Emitting Disc (JED) occupies a large zone in the accretion disc. b) Hard state with much larger $\dot{m}$ and smaller $r_{J}$ : the pair creation threshold is still not reached. c) Soft state when $\dot{m}$ is such that there is no zone anymore within the disc where an equipartition field is present: no JED, hence neither MHD jet nor pair beam. d) Luminous Intermediate state between the Hard and the Soft states: the high disc luminosity (SAD) combined with the presence of a MHD jet allows pair creation and acceleration along the axis, giving birth to flares and superluminal ejection events.

Hameury et al. 1997). However, such a model does not account for jets and their radio emission, even though $\mathrm{XrBs}$ in quiescence seem also to follow the radio/X-ray correlation (e.g. Fender et al. 2003; Gallo et al. 2004, 2005).

Within our framework, a BH $\mathrm{XrB}$ in quiescence has a large $r_{J}$, so that a large zone in the whole disc is driving jets (Fig. 2a). The low $\dot{m}$ provides a low synchrotron jet luminosity, while the JED is optically thin, producing a SED probably very similar to that of an ADAF. We thus expect $r_{J} \sim r_{\text {tr }}$. The weak MHD Poynting flux prevents the ignition of the pair cascade process and no pair beam is produced.

It must be noted that slightly more complicated situations can arise depending on the actual value of $\dot{m}$. For instance, the innermost denser regions of the JED could become optically thick for larger values of $\dot{m}$, i.e closer to the Hard state level.

\subsection{The Hard state}

Within our framework, the JED is now more limited radially than in the Quiescent state, namely $r_{J} \sim 40-100 r_{\mathrm{g}}$ (Fig. 2b). This transition radius corresponds to the inner disc radius $r_{\text {in }}$ as obtained within the SAD framework (Zdziarski et al. 2004). Due to the higher $\dot{m}$, the JED may become optically thick, which is required to explain the broad iron lines observed in some binary systems (Nowak et al. 2002; Sidoli \& Mereghetti 2002; Frontera et al. 2001). The low velocity of the plasma expected at the jet basis is in good agreement with recent studies of XrBs in Hard state (Maccarone 2003; Gallo et al. 2003). It can also explain the apparent weakness of the Compton reflection (Zdziarski et al. 1999; Gilfanov et al. 1999) as already suggested by Markoff et al. (2003, see also Beloborodov 1999; Malzac et al. 2001) and tested by Markoff \& Nowak (2004). In any case, the JED intrinsic emission is weak with respect to that of the outer standard disc: most of the accretion power flows out of the JED as an MHD Poynting flux. Nevertheless, the threshold for pair creation is still not reached and there is no pair beam, hence no superluminal motion. The MHD power is therefore shared between the jet basis, whose temperature increases (the thermal "corona") producing X-rays, and the largescale jet seen as the persistent (synchrotron) radio emission.

\subsection{The Soft state}

Our interpretation of the Soft state relies on the disappearance of the JED, i.e. when $r_{J}$ becomes smaller or equal to $r_{i}$ (Fig. $2 \mathrm{c}$ ). Depending on the importance of the magnetic flux in the disc, 
this may occur at different accretion rates. Thus, the threshold in $\dot{m}$ where there is no region anymore in the disc with equipartition fields may vary. The whole disc adopts therefore a radial structure akin to the standard disc model. Since no MHD jet is produced, all associated spectral signatures disappear. Even if pair production may take place (when $\dot{m}$ is large), the absence of the confining MHD jet forbids the pairs to get warm enough and be accelerated: no superluminal motion should be detected.

Note however that the presence of magnetic fields may be the cause of particle acceleration responsible for the weak hardenergy tail (McCR03, Zdziarski \& Gierlinski 2003, and references therein).

\subsection{Intermediate states}

This state has been first identified at large luminosities $(L>$ $\left.0.2 L_{\text {Edd }}\right)$ and was initially called Very High state. However, high luminosity appeared to not be a generic feature since it has be observed at luminosities as low as $0.02 L_{\text {Edd }}$ (McCR03; Zdziarski \& Gierlinski 2003). Therefore, the most prominent feature is that these states are generally observed during transitions between Hard and Soft states. Within our framework, they correspond to geometrical situations where $r_{J}$ is small but remains larger than $r_{i}$ (Fig. 2d). The flux of the outer standard disc is thus important while the JED is occupying a smaller volume. The consequences on the spectral shape are not straightforward since the importance of the different spectral components relative to each other depends on the precise values of $r_{J}$ and $\dot{m}$. Such study is out of the scope of the present paper and will be detailed elsewhere.

The crucial point however is that, in our framework, luminous intermediate states (the so-called Very High State or VHS) with high $\dot{m}$ provide the best conditions for the formation of the ultra-relativistic pair beam, as described in details in Sect. 2.5: (1) a high luminosity; (2) a high energy steep power law spectrum extended up to the $\gamma$-ray bands; and (3) the presence of the MHD jet. The two first characteristics enable a $\gamma-\gamma$ opacity larger than unity (cf. Eq. (22) of Sect. 2.5), while the MHD jet allows to confine the pair beam and maintain the pair warm, a necessary condition to trigger a pair runaway process. The total emission would be then dominated by the explosive behavior of the pairs, with the sudden release of blobs. Each blob produced in the beam first radiates in $\mathrm{X}$ and $\gamma$-ray, explaining the hard tail present in this state, and then, after a rapid expansion, produces the optically thin radio emission. This pair beam would also explain the superluminal ejections observed during this state in different objects (e.g. Sobczak et al. 2000; Hannikainen et al. 2001). We conjecture that the exact moment where this occurs corresponds to the crossing of the "jet line" recently proposed by (Fender et al. 2004, see also Corbel et al. 2004). This corresponds to a transition from the "hard" intermediate state to the "soft" one.

The rapid increase of the pair beam pressure in the inner region of the MHD jet, during a strong outburst, may dramatically perturb the MHD jet production. Indeed, a huge pair pressure at the axis may enforce the magnetic surfaces to open dramatically, thereby creating a magnetic compression on the JED so that no more ejection is feasible. Alternatively, it is also possible that the racing of the pair process completely wears out the MHD Poynting flux released by the JED, suppressing the jet emission or even the jet itself. Whatever occurs (i.e. jet destruction or jet fading), we expect a suppression of the steady jet emission when a large outburst sets in. Interestingly, the detailed spectral and timing study of the radio/X-ray emission of four different black hole binaries during a major radio outburst (Fender et al. 2004) shows a weakening and softening of the $\mathrm{X}$-ray emission as well as a the quenching of the radio emission after the burst. This is in good agreement with our expectations since the cooling of the pair beam should indeed results in a flux decrease and a softening of its spectrum.

\section{Temporal properties}

Since both SAD and JED are quasi Keplerian, the first obvious time scale is the Keplerian orbit time, namely

$\tau_{\mathrm{D}}\left(r_{J}\right)=\frac{2 \pi}{\Omega_{\mathrm{k}}}=0.1\left(\frac{m}{10}\right)\left(\frac{r_{J}}{50 r_{\mathrm{g}}}\right)^{3 / 2} \mathrm{~s}$

measured here at the transition radius $r_{J}$. Since gravity is the dominant force, this time is also the dynamical time involved whenever physical conditions are locally modified within the disc. This time scale is much smaller than the duration of a spectral state or even the transition between two states (but comparable to some timing features, see below). In fact, the time evolution of $\mathrm{BH} \mathrm{XrBs}$ requires large variations of the disc accretion rate $\dot{m}$. The time scales involved, namely rising and decay times but also periodicity, if any, depend therefore on conditions at the outer accretion disc. The inner disc region will thus respond to these variations with its own time scales, which introduces a delay but more importantly a temporal convolution (it acts as a filter). We do dot intend to address the issue of the timing behavior of $\mathrm{BH}$ XrBs. As discussed earlier, this requires, within our framework, to take into account the evolution of the large scale magnetic field. Here, we just remark that the presence of these four dynamical components (SAD, JED, MHD jet and pair beam) introduces interesting temporal properties that may be relevant to observations.

Let us assume an increase in $\dot{m}$ triggered at some outer radius $r_{\text {out }} \gg r_{J}$. This information propagates towards the center with the accretion flow, as a front of increased total pressure. The time scale involved is therefore $\tau_{\text {acc,SAD }}\left(r_{\text {out }}\right) \sim r_{\text {out }} / u_{r}$. If we assume that $r_{J}$ decreases (because of a decrease in $\mu$ ), then the JED (and its associated MHD jets) will progressively disappear. This evolution from a Hard state to an Intermediate or Soft state will be controlled by the advance of this front, namely

$\tau_{\mathrm{acc}, \mathrm{SAD}} \simeq 170 \alpha_{\mathrm{v}}^{-1}\left(\frac{\varepsilon}{0.01}\right)^{-2}\left(\frac{m}{10}\right)\left(\frac{r_{J}}{50 r_{\mathrm{g}}}\right)^{3 / 2} \mathrm{~s}$.

If, due to a change in $\dot{m}$, the radial distribution of the vertical magnetic field needs to be readjusted, then this is done quite fast. Indeed, within the SAD, the time scale for field diffusion is the accretion time scale, since $\tau_{\text {diff }}=r^{2} / \nu_{\mathrm{m}} \sim \tau_{\mathrm{acc}} \mathcal{R}_{\mathrm{m}}$ with $\mathcal{R}_{\mathrm{m}} \sim 1$. Inside the JED, the diffusion time scale is much longer than the accretion time scale because $\mathcal{R}_{\mathrm{m}} \sim \varepsilon^{-1}$ 
(Ferreira \& Pelletier 1995). However, the accretion time scale inside the JED is itself much shorter than in the SAD (see Eq. (11)), so that

$\tau_{\text {diff,JED }} \simeq \alpha_{\mathrm{v}} \tau_{\mathrm{acc}, \mathrm{SAD}}$.

Another interesting timing feature is introduced by the MHD jets launched from the JED. Indeed, any adjustment in the disc leads inevitably to a modification of the jet parameters, e.g. the ejection efficiency $\xi$. The time scale for this readjustment can be considered to be of the order of the travel time of the fast MHD waves, namely

$\tau_{\text {jet }}(r) \simeq \int_{h}^{s_{\mathrm{FM}}} \frac{\mathrm{d} s}{V_{\mathrm{FM}}}$

where $V_{\mathrm{FM}}$ is the speed of the fast magnetosonic wave and the integration is made along a magnetic surface anchored at a disc radius $r$ (from the disc surface $h$ to the fast point $s_{\mathrm{FM}}$ ). We computed this time using the MAES solutions of Ferreira (1997) and illustrated in his Fig. 6. Solutions crossing the fast point right after the Alfvén point (larger $\xi$ ) display $\tau_{\text {jet }}(r) \sim \tau_{\mathrm{D}}(r)$ but undergo a recollimation shock right after that. Jet solutions with smaller $\xi$ propagate much farther away and have $\tau_{\text {jet }}(r) \sim 10^{2} \tau_{\mathrm{D}}(r)$. It is not clear yet if these time scales provide an explanation to some observed timing properties. But the fact that jets are indeed observed is a strong indication that one should take into account their dynamics.

In our description of the canonical spectral states of $\mathrm{XrBs}$ we did not mention the important issue of quasi-periodic oscillations or QPOs. Low-frequency $(0.1-30 \mathrm{~Hz})$ QPOs in the $\mathrm{X}$-ray bands $(2-30 \mathrm{keV})$ are indeed observed in the Hard and luminous Intermediate (VHS) states. In the latter state, higher frequencies (up to $300 \mathrm{~Hz}$ ) are also present (e.g. Remillard et al. 2002; McCR03). We do not offer yet any precise explanation to these phenomena. However, we note that QPOs are stronger in the hard X-ray bands ( 20-30 keV; e.g. Rodriguez et al. 2004) and are correlated with the radio flux (Migliari et al. 2005). Their emission must then be related to the dynamics involved in the ejection events (both steady and eruptive).

Interestingly, our framework provides a promising environment for the onset of instabilities leading to QPOs. The inner pair beam is an intermittent flow from the inner regions (several $r_{i}$ ) and could therefore be responsible for some of the high frequency QPOs. On the other hand, the MHD jet may provide low frequency QPOs. For instance, if some disc material, continuously ejected just outside $r_{J}$, is failing to become superAlfvénic, then one would expect waves going back and forth between the disc surface and the Alfvén surface (located at $r_{\mathrm{A}}$ and $z_{\mathrm{A}}$ in cylindrical coordinates) where a shock is occurring. A crude estimate of the frequency gives $v \sim V_{\mathrm{A}} / z_{\mathrm{A}} \sim \Omega_{*} r_{\mathrm{A}} / z_{\mathrm{A}} \sim$ $\Omega_{\mathrm{k}}\left(r_{J}\right)$ since $\Omega_{*} r_{\mathrm{A}} \sim V_{\mathrm{A}}$ and $z_{\mathrm{A}} \sim r_{\mathrm{A}}$ in magnetically driven jets (Ferreira 1997) and $\Omega_{*}=\Omega_{\mathrm{k}}\left(r_{J}\right)$ is the rotation rate of the magnetic surface. For a radius $r_{J} \sim 50 r_{\mathrm{g}}$, this gives a $\sim 10 \mathrm{~Hz}$ QPO. This clearly deserves further investigation.

\section{Summary and concluding remarks}

We present in this paper a new paradigm for the accretionejection properties of Galactic Black Hole X-ray binaries. We assume the existence of a large scale magnetic field of bipolar topology in the innermost disc regions. Such a field allows for several dynamical phenomena to occur whose relative importance determine the observed spectral state of the binary. The dynamical constituents are: (1) an outer standard accretion disc (SAD) for $r>r_{J}$; (2) an inner Jet Emitting Disc (JED) for $r<r_{J}$ driving; (3) a self-collimated non-relativistic electronproton surrounding, when adequate conditions are met; (4) a ultra-relativistic electron-positron beam. The dynamical properties of each constituent have been thoroughly analyzed in previous works (e.g. Shakura \& Sunyaev 1973; Henri \& Pelletier 1991; Ferreira \& Pelletier 1995; Marcowith et al. 1997; Renaud \& Henri 1998; Saugé \& Henri 2003, 2004), but it is the first time where they are invoked altogether as necessary ingredients to reproduce the different spectral states of a same object.

We showed that the various canonical states can be qualitatively explained by varying independently the transition radius $r_{J}$ and the disc accretion rate $\dot{m}$. In our view, the Quiescent and Hard states are dominated by non relativistic jet production from the JED, providing henceforth a persistent synchrotron jet emission. The Soft state is obtained when the transition radius $r_{J}$ becomes smaller than the last marginally stable orbit $r_{i}$, a SAD is established throughout the whole accretion disc. Intermediate states, between Hard and Soft, are expected to display quite intricate and variable spectral energy distributions. Luminous Intermediate states, obtained during the Hardto-Soft transitions, are those providing the unique conditions for intermittent pair creation. These pairs give rise to a ultra relativistic beam propagating on the MHD jet axis, explaining both the observed superluminal motions and hard energy tail.

The dynamical structure presented here (JED, SAD, MHD jet and, occasionally, a pair beam) seems to be consistent with all available information about the canonical spectral states of $\mathrm{BH} \mathrm{XrBs}$. However, a more quantitative analysis is critical. In particular, we need to show that the base of the MHD jet can indeed provide a hot corona with the correct spectral signature. Then, a precise estimate of the radio/X-ray correlation predicted by our model and its comparison to observations will be a test of prime importance for its validity. This is postponed to a future work (Petrucci et al., in preparation).

In our view, the magnetic flux available at the inner disc regions is a fundamental and unavoidable ingredient that most probably varies from one system to another. Since changing the amount of magnetic flux changes the transition radius $r_{J}$, the characteristic value of $\dot{m}$ (hence luminosity) associated with each spectral state is also modified. Also, if accreting material is carrying magnetic flux of opposite direction (Tagger et al. 2004), then this should lead to a major readjustment of the whole magnetic structure. Clearly, taking into account the advection of a large scale magnetic field within the disc introduces a whole new set of variable phenomena.

Finally, we note that the typical values of the magnetic field required to steadily launch jets from JEDs, given in Eq. (13), are consistent with observational estimates (Gliozzi et al. 1999; Gnedin \& Natsvlishvili 1997; ?). We expect that $\mathrm{BH} \mathrm{XrBs}$ should radiate above the $\mathrm{MeV}$ range during luminous intermediate states, when pairs are produced. A similar proposal has been developed recently by 
Bosch-Ramon \& Paredes (2004a,b) but it is here a natural outcome of our model. Very interestingly, such a high energy emission has been recently detected by the HESS instrument in the $\mathrm{TeV}$ range (Aharonian et al. 2005). Note also that two microquasars were already detected by EGRET, LS 5039 (Paredes et al. 2000) and LS I +61 303 (Massi et al. 2004, for a recent review), and there is possibly other unidentified galactic EGRET sources (Paredes 2004). Besides, the $\gamma$-ray emission of the pair beam can occur further away along the jet at the $\gamma$ ray photosphere, as proposed for AGNs (e.g. Marcowith et al. 1995). Noticeably, $\gamma$-ray spectra of the possible EGRET counterparts seem to exhibit a break in the $\mathrm{MeV}$ range, very similar to that observed in many AGNs. This break could be explained by the transition from the optically thin X-ray component to the optically thick, photosphere dominated, $\gamma$-ray component. This prediction of $\gamma$-ray emission of microquasars during very high flaring states could be tested by future GLAST observations.

Acknowledgements. We thank S. Corbel for a careful reading of the manuscript and T. Belloni for having sent us a draft of its paper before it was completely accepted.

\section{References}

Aharonian, F., Akhperjanian, A. G., Aye, K.-M., et al. 2005, Science, 309, 746

Balbus, S. A., \& Hawley, J. F. 1991, ApJ, 376, 214

Belloni, T., Homan, J. P. C., Cassella, P., et al. 2005 [arXiv: astro-ph/0504577]

Belloni, T., Mendez, M., King, A. R., van der Klis, M., \& van Paradijs, J. 1997, ApJ, 479, L145

Beloborodov, A. M. 1999, in High Energy Processes in Accreting Black Holes, ASP Conf. Ser., 161, 295

Blandford, R. D., \& Begelman, M. C. 1999, MNRAS, 303, L1

Blandford, R. D., \& Begelman, M. C. 2004, MNRAS, 349, 68

Bogovalov, S., \& Tsinganos, K. 2001, MNRAS, 325, 249

Bogovalov, S. V. 2001, A\&A, 371, 1155

Bosch-Ramon, V., \& Paredes, J. M. 2004a, A\&A, 417, 1075

Bosch-Ramon, V., \& Paredes, J. M. 2004b, A\&A, 425, 1069

Buxton, M. M., \& Bailyn, C. D. 2004, ApJ, 615, 880

Casse, F., \& Ferreira, J. 2000a, A\&A, 353, 1115

Casse, F., \& Ferreira, J. 2000b, A\&A, 361, 1178

Choudhury, M., Rao, A. R., Vadawale, S. V., \& Jain, A. K. 2003, ApJ, 593, 452

Corbel, S., Fender, R. P., Tzioumis, A. K., et al. 2000, A\&A, 359, 251

Corbel, S., Nowak, M. A., Fender, R. P., Tzioumis, A. K., \& Markoff, S. 2003, A\&A, 400, 1007

Corbel, S., Fender, R. P., Tomsick, J. A., Tzioumis, A. K., \& Tingay, S. 2004, ApJ, 617, 1272

Dhawan, V., Mirabel, I. F., \& Rodríguez, L. F. 2000, ApJ, 543, 373

Esin, A. A., McClintock, J. E., \& Narayan, R. 1997, ApJ, 489, 865

Esin, A. A., Narayan, R., Cui, W., Grove, J. E., \& Zhang, S.-N. 1998, ApJ, 505, 854

Esin, A. A., McClintock, J. E., Drake, J. J., et al. 2001, ApJ, 555, 483

Falcke, H., \& Biermann, P. L. 1995, A\&A, 293, 665

Falcke, H., Körding, E., \& Markoff, S. 2004, A\&A, 414, 895

Fender, R., \& Belloni, T. 2004, ARA\&A, 42, 317

Fender, R., Corbel, S., Tzioumis, T., et al. 1999, ApJ, 519, L165

Fender, R. P., Gallo, E., \& Jonker, P. G. 2003, MNRAS, 343, L99
Fender, R. P., Belloni, T. M., \& Gallo, E. 2004, MNRAS, 355, 1105

Ferreira, J. 1997, A\&A, 319, 340

Ferreira, J. 2002, in Star Formation and the Physics of Young Stars, ed. J. Bouvier, \& J.-P. Zahn (EAS Publications Series), 3, 229 [arXiv:astro-ph/0311621]

Ferreira, J., \& Casse, F. 2004, ApJ, 601, L139

Ferreira, J., \& Pelletier, G. 1993, A\&A, 276, 625

Ferreira, J., \& Pelletier, G. 1995, A\&A, 295, 807

Frontera, F., Palazzi, E., Zdziarski, A. A., et al. 2001, ApJ, 546, 1027

Galeev, A. A., Rosner, R., \& Vaiana, G. S. 1979, ApJ, 229, 318

Gallo, E., Fender, R. P., \& Pooley, G. G. 2003, MNRAS, 344, 60

Gallo, E., Corbel, S., Fender, R. P., Maccarone, T. J., \& Tzioumis, A. K. 2004, MNRAS, 347, L52

Gallo, E., Fender, R. P., \& Hynes, R. I. 2005, MNRAS, 356, 1017

Gilfanov, M., Churazov, E., \& Revnivtsev, M. 1999, A\&A, 352, 182

Gliozzi, M., Bodo, G., \& Ghisellini, G. 1999, MNRAS, 303, L37

Gnedin, Y. N., \& Natsvlishvili, T. M. 1997, in Stellar Magnetic Fields, Proceedings of the International Conference, held in the Special Astrophysical Observatory of the Russian AS, May 13-18, 1996, ed. Yu. Glagolevskij, \& I. Romanyuk (Special Astrophysical Observatory Press), 40

Grove, J. E., Johnson, W. N., Kroeger, R. A., et al. 1998, ApJ, 500, 899

Haardt, F. 1993, ApJ, 413, 680

Hameury, J.-M., Lasota, J.-P., McClintock, J. E., \& Narayan, R. 1997, ApJ, 489, 234

Hannikainen, D., Campbell-Wilson, D., Hunstead, R., et al. 2001, ApSSS, 276, 45

Heinz, S. 2004, MNRAS, 355, 835

Heinz, S., \& Sunyaev, R. A. 2003, MNRAS, 343, L59

Henri, G., \& Pelletier, G. 1991, ApJ, 383, L7

Heyvaerts, J. F., \& Priest, E. R. 1989, A\&A, 216, 230

Königl, A. 2004, ApJ, 617, 1267

Kalemci, E., Tomsick, J. A., Buxton, M. M., et al. 2005, ApJ, 622, 508

King, A. R., Pringle, J. E., West, R. G., \& Livio, M. 2004, MNRAS, 348, 111

Lasota, J.-P., Narayan, R., \& Yi, I. 1996, A\&A, 314, 813

Livio, M., Pringle, J. E., \& King, A. R. 2003, ApJ, 593, 184

Maccarone, T. J. 2003, A\&A, 409, 697

Mahadevan, R. 1997, ApJ, 477, 585

Malzac, J., Beloborodov, A. M., \& Poutanen, J. 2001, MNRAS, 326, 417

Marcowith, A., Henri, G., \& Pelletier, G. 1995, MNRAS, 277, 681

Marcowith, A., Pelletier, G., \& Henri, G. 1997, A\&A, 323, 271

Marcowith, A., Henri, G., \& Renaud, N. 1998, A\&A, 331, L57

Markoff, S. 2004, in X-ray Binaries to Quasars: Black Hole Accretion on All Mass Scales, ed. T. J. Maccarone, R. P. Fender, \& L. C. Ho

Markoff, S., Falcke, H., \& Fender, R. 2001, A\&A, 372, L25

Markoff, S., Nowak, M., Corbel, S., Fender, R., \& Falcke, H. 2003, A\&A, 397, 645

Markoff, S., \& Nowak, M. A. 2004, ApJ, 609, 972

Massi, J. M., Ribo, M., Paredes, J. M., et al. 2004, in AIP Proc. Ser. [arXiv: astro-ph/0410504]

McClintock, J. E., \& Remillard, R. A. 2003 [arXiv: astro-ph/0306213] (McCR03)

Merloni, A., \& Fabian, A. C. 2002, MNRAS, 332, 165

Merloni, A., Heinz, S., \& di Matteo, T. 2003, MNRAS, 345, 1057

Migliari, S., Fender, R. P., \& Van der Klis, M. 2005 [arXiv: astro-ph/0507223]

Mirabel, I. F., \& Rodriguez, L. F. 1998, Nature, 392, 673

Mirabel, I. F., \& Rodríguez, L. F. 1999, ARA\&A, 37, 409

Narayan, R., McClintock, J. E., \& Yi, I. 1996, ApJ, 457, 821

Nowak, M. A., Wilms, J., \& Dove, J. B. 2002, MNRAS, 332, 856 
O’Dell, S. L. 1981, ApJ, 243, L147

Paredes, J. M. 2004, in V Microquasar Workshop, Beijing, June 2004 [arXiv: astro-ph/0409226]

Paredes, J. M., Martí, J., Ribó, M., \& Massi, M. 2000, Science, 288, 2340

Pelletier, G. 2004, in Dynamics and dissipation in electromagnetically dominated media (Nova Science), ed. M. Lyutikov [arXiv: astro-ph/0405113]

Pelletier, G., \& Roland, J. 1989, A\&A, 224, 24

Pelletier, G., \& Sol, H. 1992, MNRAS, 254, 635

Pelletier, G., Sol, H., \& Asseo, E. 1988, Phys. Rev. A, 38, 2552

Petrucci, P. O., Haardt, F., Maraschi, L., et al. 2000, ApJ, 540, 131

Phinney, E. S. 1987, in Superluminal Radio Sources, 301

Pietrini, P., \& Krolik, J. H. 1995, ApJ, 447, 526

Poutanen, J., \& Svensson, R. 1996, ApJ, 470, 249

Remillard, R. A., Sobczak, G. J., Muno, M. P., \& McClintock, J. E. 2002, ApJ, 564, 962

Renaud, N., \& Henri, G. 1998, MNRAS, 300, 1047

Robertson, S. L., \& Leiter, D. J. 2004, MNRAS, 350, 1391

Rodriguez, J., Corbel, S., Hannikainen, D. C., et al. 2004, ApJ, 615, 416

Romanova, M. M., Ustyugova, G. V., Koldoba, A. V., Chechetkin, V. M., \& Lovelace, R. V. E. 1998, ApJ, 500, 703

Saugé, L., \& Henri, G. 2003, New Astron. Rev., 47, 529
Saugé, L., \& Henri, G. 2004, ApJ, 616, 136

Shakura, N. I., \& Sunyaev, R. A. 1973, A\&A, 24, 337

Sidoli, L., \& Mereghetti, S. 2002, A\&A, 388, 293

Sobczak, G. J., McClintock, J. E., Remillard, R. A., et al. 2000, ApJ, 544, 993

Sol, H., Pelletier, G., \& Asseo, E. 1989, MNRAS, 237, 411

Stirling, A. M., Spencer, R. E., de la Force, C. J., et al. 2001, MNRAS, 327,1273

Stone, J. M., Hawley, J. F., Gammie, C. F., \& Balbus, S. A. 1996, ApJ, 463, 656

Tagger, M., Varnière, P., Rodriguez, J., \& Pellat, R. 2004, ApJ, 607, 410

Tanaka, Y., \& Lewin, W. H. G. 1995, in X-Ray Binaries, ed. W. H. G. Lewin, J. van Paradijs, \& E. P. J. van den Heuvel (Cambridge: Cambridge Univ. Press), 126

Vadawale, S. V., Rao, A. R., \& Chakrabarti, S. K. 2001, A\&A, 372, 793

Zdziarski, A. A., \& Gierlinski, M. 2003, in Proc. of Stellar-mass, intermediate-mass, and supermassive black holes, Kyoto (ZG04), 99 [arXiv: astro-ph/0403683]

Zdziarski, A. A., Lubinski, P., \& Smith, D. A. 1999, MNRAS, 303, L11

Zdziarski, A. A., Gierliński, M., Mikołajewska, J., et al. 2004, MNRAS, 351, 791 